\title{
Evolution de la société et évolution de la formation de l'ingénieur hydraulicien
}

\author{
Claude Thirriot \\ E.N.S.E.E.I.H.T.
}

\section{Introduction}

La sécheresse, la pollution des nappes et des rivières ont attiré de manière médiatique l'attention sur les métiers de l'eau. L'étude en soufflerie numérique des avions puis des maquettes d'automobile ont fait glisser le champ des mécaniciens des fluides du tube de Pitot à l'ordinateur vectoriel. La spirale de l'irrigation et de l'investissement hydroagricole transforme l'hydropédologue en économiste cernant les aléas de la météorologie turbulente de la production laitière ou céréalière engoncée dans les garde-fous européens face aux soubresauts des cours mondiaux. L'hydroludique prend des parts de marché dans le PIB des loisirs en développement exponentiel. La recherche aspire ou attire ou abrite les scientifiques décontenancés voire effrayés par la compétition internationale du productivisme.

L'enseignement et la formation des ingénieurs hydrauliciens peuvent-ils rester indifférents aux mutations de société commencées hier et constatées aujourd'hui et plus encore en germe imperceptible maintenant et qui modifieront demain le comportement technique et éthique d'ingénieurs-citoyens dans les domaines stratégiques de l'eau et de l'énergie?

Le propos peut paraître bien ambitieux et même déplacé chez un professeur de mécanique des fluides, car on l'attend et l'entend plutôt d'un sociologue. Mais puisque nous faisons ici de la futurologie, débridons le temps de quelques lignes notre réserve scientifique pour parler de la convolution de l'étudiant d'aujourd'hui et de l'ingénieur hydraulicien de demain.

\section{Analyse des systèmes et structure de production pédagogique}

La mission de nos structures de formation d'ingénieur hydraulicien fonctionne comme tout système rencontré en régulation fluidique ou pneumatique. Une donnée à l'entrée, ici un échantillon d'étudiants est transformée par une fonction de transfert, ici à caractère pédagogique, en un résultat ou effet à la sortie, ici une promotion d'ingénieurs hydrauliciens. Rien de plus classique, c'est tout à fait analogue à ce qui se passe dans la relation pluie-débit avec la fonction de production, à ceci près qu'on a des exigences sur la sortie et qu'on croit pouvoir jouer sur la fonction de transfert pédagogique alors que l'hydrologue avoue son impuissance, ou presque, à maîtriser la fonction production imposée par la nature (sauf dans les villes où l'homme s'ingénie par son vertige mégalopolique à compliquer et détériorer cette fonction production sublimée en assainissement).

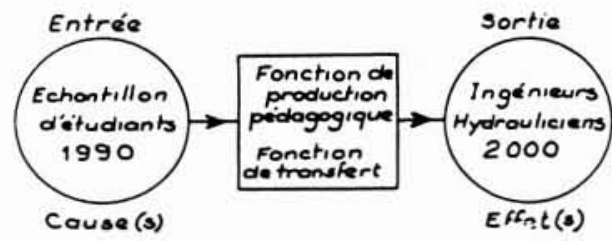

Mais dans le fond, le schéma de la fonction de production hydrologique est plus proche de la chaîne pédagogique que le système entrée-sortie de l'automatique et ceci surtout par le flou dû à l'imprécis et à l'aléatoire.

Pour paraître à la mode, on parle aujourd'hui beaucoup d'algèbre des ensembles flous (fuzzy sets) et de théorie des possibilités, c'est peut être avec ce langage mathématique qu'il faudrait systématiser notre propos de formation mais nous en resterons cependant à l'approche concrète de l'électeur moyen ou même mieux du contribuable moyen qui souhaiterait mieux comprendre comment fonctionne le tonneau des Danaïdes d'un ministère de l'Education nationale.

Les sous sont mis dans la fonction de transfert mais la sortie est-elle bien caractérisée ? Sait-on clairement quel est le portrait robot de l'ingénieur hydraulique de l'an 2000 ? Là intervient déjà une première fonction retard. Indécis sur les vrais besoins de la société en 2000 , on va caler le programme pédagogique sur notre regard actuel sur notre société actuelle ou au mieux on précèdera par une extrapolation exponentielle (dont on sait tout le bien pour le programme de suréquipement nucléaire d'EDF face à la prévision de consommation énergétique). 


\section{Les objectifs : l'ingénieur hydraulicien de demain en nombre et en qualité}

C'est bien connu, la France est sous développée du point de vue de la production d'ingénieurs. A peu près 30000 ingénieurs formés par an en Grande-Bretagne et en République Fédérale Allemande (que sera-ce avec la réunification!) à peine 14000 par an en France.

Un des premiers objectifs pourrait donc être quantitatif. Doublons la production annuelle d'ingénieurs hydrauliciens, ils s'adapteront ensuite dans les bureaux de calcul informatique, dans l'automobile et l'agroalimentaire, voire dans la banque. Le discours peut paraitre ironique mais au deuxième degré, il n'est pas complètement dénué de sens. Avec le vernier pédagogique que constitue la formation continue, il faut mieux un ingénieur hydraulicien pour gérer les approvisionnements d'un supermarché que pas de cadre technique du tout. Mais l'avenir de nos ingénieurs hydrauliciens n'est pas uniquement dans le hamburger de chez Mc Donald. Certes la Société est et semble vouloir rester un temps encore de consommation, mais résolument optimiste, on peut penser qu'il y aura une évolution du menu. Et déjà, on achoppe sur une question capitale : les objectifs sociologiques de la formation de nos ingénieurs sont-ils définis de manière extrinsèque et imposés à l'outil enseignant ou bien peut-il déjà apparaître un subtil effet de contreréaction (au sens cybernétique bien sûr) et la formation même en établissement scientifique peut-elle infléchir sur ces objectifs, c'est-à-dire sur les besoins de la société de demain qui risquent d'être pour partie un superbe fruit médiatique irrésistible par son aspect, fade à l'intérieur ? Didactique et éthique? On reviendra sur la question, on s'en doute.

Quels seront donc les besoins en l'An 2000 ? Plus d'aqualands parce qu'on ne peut plus se baigner dans les rivières de ma jeunesse? Deux ou trois réseaux d'eau côte à côte dans les villes? Un filet précieux pour l'eau alimentaire garantie à prix d'or (noir) sans nitrates. De l'eau moyennement traitée pour les besoins domestiques, une eau d'irrigation des pelouses avec les additions rationnelles en engrais dont l'utilisation sera par ailleurs sévèrement contrôlée par la loi, le premier labyrinthe souterrain du transport des eaux usées et des suspensions, le second réseau séparé de l'assainissement pluvial lui même avec des parcs de contrôle pour éviter à des dizaines de tonnes de poissons de servir d'indicateur d'orageur lessiveur, le ventre en l'air, encore un treillis de canaux urbains où circuleront les coches d'eau pour laisser aux piétons et aux cyclistes les rues sans pollution automobile des villes futures ? Et de six pour trois réseaux annoncés! « Le Meilleur des Mondes ", « Soleil Vert» ou « Ravages ».

Bien sûr, il est hors de mon propos d'esquiver par une pirouette ironique le fond du problème de l'objectif : des ingénieurs hydrauliciens, pour quoi faire en l'An 2000 ? Pour animer le débat, je proposerai trois missions qui pourront parfois s'imbriquer dans la vie d'un même homme, ou correspondre à trois tempéraments différents. Ce sont la vestale de l'eau, le promoteur, le chercheur.

La vestale de l'eau a d'abord les tâches ancillaires de fournir à ses concitoyens une eau pure et agréable au palais et d'assurer l'environnement aquatique propre au plaisir de la vie certes, mais d'abord à la vie tout court. Dans cette mission vieille comme le monde industriel, rien de changé par rapport aux exigences imposées à l'ingénieur hydraulicien d'aujourd'hui mais pourquoi vouloir changer à tout prix pour faire plus mal. Il faudra encore dans cinquante ans des ingénieurs des services urbains des eaux qui soient consciencieux, compétents et conviviaux. Il faudra des ingénieurs des services agricoles soucieux à la fois des intérêts des agriculteurs et de la protection de la ressource hydrique aussi bien aux points de vue de la quantité que de la qualité. L'écolo doux et rêveur a maintenant droit de cité et doit se métamorphoser en ingénieur actif, efficace mais sereinement conscient. Même le Président Mitterrand, dans les flonflons du 14 juillet a célébré l'avènement écologique. "Le mérite des écologistes, c'est d'avoir pressenti avant les autres que les problèmes de la nature, de la relation entre l'homme et la nature, de la protection de l'environnement étaient une donnée majeure de l'époque industrielle qui est la nôtre... Alors je rends hommage aux écologistes, ils ont vu juste. Maintenant dans la pratique des choses, moi je suis là pour aider tous ceux qui se battront pour la sauvegarde de la nature" (Le Monde).

Le promoteur est celui par qui le produit nouveau arrive. Il n'est pas question sous raison juste et profonde de défense écologique, d'interdire l'inventivité mais seulement de bien contrôler les effets. Quels seront les produits nouveaux hydrauliques en 2000 ? Dans le fond, est-ce mon manque d'imagination mais je ne me sens pas viscéralement concerné par cet aspect du problème. Je ne dois pas avoir les appétits d'un commerçant. Bien sûr l'eau, la mécanique des fluides et l'énergie auront bien des choses à créer ensemble. Mais l'hydraulique même très élargie à la mécanique des fluides paraît bien pauvrette par rapport à la physique de la matière qui a engendré aussi bien la circulation électronique des circuits informatiques que les multiples visages des matériaux plastiques. Alors pour une part l'avenir de l'hydraulicien est dans la coopération avec des secteurs actuellement plus créateurs.

Le chercheur enfin. Mais chercher quoi et pourquoi? D'abord pour lui-même. Dans une société nantie et repue, où les machines feront pour une grande part mieux et plus vite que l'homme, la recherche peut être un passe temps ludique et personnifiant. Comme celui d'aujourd'hui, le chercheur de demain trouvera dans son activité une exaltation un peu narcissique qui vaut bien quelque sacrifice sur le salaire quand le pain et le logis sont, tout de même, assurés. Bien sûr, il n'est tout de même pas interdit au chercheur de trouver, de trouver même des choses matérielles ou conceptuelles utiles à son voisin. Mais à mon avis et à mon expérience, le but de la recherche restera au moins à quatre-vingt pour cent le plaisir profond du chercheur, plaisir dont des miettes pourront égayer ses semblables tout comme les arpèges d'un pianiste de concert ravissent les oreilles d'aficionados ou de béotiens. 


\section{Portrait robot de l'étudiant hydraulicien de l'an 2000}

« La prévision est une opération toujours délicate surtout s'il s'agit de l'avenir." Ce trait d'humour doit rendre prudent si l'on essaie de dessiner le comportement de l'étudiant dans dix ans. Plus que l'extrapolation, il faudrait utiliser la théorie des catastrophes chère à ce philosophe mathématicien qu'est René Тном et construire quelques scénarios. Cet étudiant de l'An 2000 ira-t-il dans le sens de l'individualisme souriant et tolérant, ignorant du corset moral de ma jeunesse que fondait la solidarité entre générations. A part quelques mystiques, pour la plupart des jeunes, la vie est là, avec sa profusion et sa diversité. "Carpe diem » et presque « no future » (sauf professionnel pour les élèves ingénieurs d'aujourd'hui qu'on s'arrache avant la sortic de l'Ecole).

L'Ecole est le passage obligé que l'on essaie de rendre le moins inconfortable possible, modulant l'effort quotidien sur le calcul des notes obtenues et l'anticipation de celles à venir. Et demain? Par suite de l'oscillation du balancier psychosociologique retrouvera-t-on une austérité à la japonaise d'hier, avec les jeunes samouraïs du savoir scientifique?

Mais telle quelle, la situation conserve des ressources énormes de créativité, d'initiative, de responsabilité, trop muselées par les formes pédagogiques traditionnelles, par dessus le marché avachies par la démagogie.

On peut penser que nos promotions avec une cinquantaine d'èlèves ingénieurs hydrauliciens sont trop peu nombreuses pour constituer des échantillons suffisamment représentatifs de la population de la jeunesse estudiantine. Certes, il y aura d'une année à l'autre des nuances de comportement apportées souvent par la personnalité des meneurs naturels. Mais j'ai été surpris de retrouver assez bien le portrait robot de nos étudiants dans un livre tout récent «Fin de siècle, début de vie, voyage au pays de 18-25 ans " (mars 1990), fruit d'une enquête de deux sociologues Daniele LINHART et Anna MALAN auprès de jeunes qui n’étaient pas étudiants pour la plupart. "Caisse de résonance de la société et groupe fortement hétérogène qui paie son tribut à la loi de la reproduction sociale, les jeunes ont done en commun des attitudes qui les distinguent des jeunes des années soixante: pragmatisme, individualisme et tolérance. " Nous retrouvons bien là les attitudes quotidiennes de nos étudiants qui recherchent le concret même dans le contenu des cours, et qui sont dans l'ensemble assez peu sensibles à la beauté des théories dont ils n'entrevoient pas l'application quasi immédiate dans leur métier. Attitude au demeurant fort utile à l'ingénieur soucieux d'efficacité mais vit-on sculement d'efficacité?

Peu embarrassés de guides idéologiques, nos étudiants sont aussi peu enclins au bénévolat, encore que quelquesuns se lancent avec enthousiasme et brio dans des activités para-universitaires comme le théâtre. Ceux-là ne sont-ils pas le signe infime de ce que seront en l'An 2000 leurs successeurs qui auront retrouvé le goût de la culture, du gratuit et des associations?
Minuscule signe d'individualisme indéniable, le fait que les travaux de préparation des mémoires de diplôme d'ingénieur longtemps réalisés en binôme soient maintenant menés en solitaire.

Peut être aussi fruit de l'individualisme, la tolérance séduisante a priori mais qui n'est peut-être qu'apathie devant ce qu'on ne peut changer sans effort communautaire et indifférence devant les autres tant que leur comportement ne nous gêne pas profondément. Quand on n'a pas de convictions fortement trempées (conviction pouvant être illusion de la vérité), la pensée des autres ne nous choque guère. "La tolérance n'est grande que quand au milieu des partis, elle est un héroïsme " écrivait Romain Rolland dans Jean-Christophe (1904-1912). L'héroïsme? Connais pas pourraient répondre dans leur presque unanimité nos élèves. Il ne s'agit pas de décider ici s'ils ont tort ou raison mais d'étiqueter les faits et traits de caractère.

Ce qu'ils souhaitent ce sont des règles clairement affichées en début d'année à partir desquelles leur système expert intuitif va définir l'optimisation de l'effort modéré pondéré par les coefficients des matières pour aller sans encombre jusqu'au diplôme.

Et demain? L'amélioration du parcours pédagogique peut venir de la stimulation du travail personnel assez de connivence avec leur individualisme mais qui sera aussi l'expansion de leur créativité et de leur besoin profond et inconscient d'initiative.

\section{Le moule pédagogique : culture et métier}

Faudra-t-il tout savoir sur l'eau depuis la physique des particules jusqu'à la description détaillée technologique des catalogues de pompes multiétages pour être le parfait ingénieur hydraulicien de l'An 2000 ? Non certainement pas, demain comme aujourd'hui tout comme du temps de Montaigne "Mieux vaut tête bien faite que tête bien bourrée. " Parce que cette tête bien faite sera la garantie de l'adaptation au changement sociologique et aux innovations technologiques, grandement aidée dans son aggiornamento scientifique ou technique par la formation continue.

Demain comme aujourd hui les maîtres mots de la pédagogie de l'ingénieur hydraulique seront culture et métier. "La culture c'est ce qui reste lorsqu'on a tout oublié » disait avec humour Edouard HERRIOT, un grand baryton du Parti Radical Socialiste de la mi-siècle. La culture, c'est l'humus de l'intelligence, c'est la ressource inconsciente de l'analogie, de la gestation de l'idée personnelle et parfois inventrice sur le riche compost des idées des autres qui ont sédimenté dans le cerveau et petit à petit crée le comportement intellectuel original de chaque personne. Le métier, c'est l'efficacité, c'est le système expert implicite personnel fait de rationnalité dans l'action et des réflexes dus à l'expérience.

Il est évidemment hors de propos d'opposer culture et métier dans la pédagogie de l'ingénieur mais tout au contraire d'assurer leur mariage heureux dans l'ubiquité de l'homo sapiens et de l'homo faber. Le dosage de pensée et action variera avec les personnes, l'important est que 
chacun étudiant et enseignant soit convaincu de l'impérative présence des deux composants dans la personnalité de l'ingénieur en général et de l'ingénieur hydraulicien en particulier.

Dans les programmes pédagogiques en école d'hydraulique, très subjectivement pour moi, cela conduit à la dichotomie entre l'essentiel et l'existentiel.

Que sera l'essentiel pour un futur ingénieur hydraulicien ? Ce qui constitue le socle cristallin de sa formation caractérisé par les trois piliers de sa connaissance: les mathématiques, l'informatique et la mécanique des fluides.

Les mathématiques seront de tous temps, c'est à la fois, un exercice spirituel et un outil pragmatique qui permet de trouver l'universalité ou l'unité dans la luxuriance de la réalité naturelle ou industrielle. " Le langage de la nature est la mathématique " disait déjà Galilée (1564-1642). Et on n'apprend bien les mathématiques qu'à vingt ans, l'âge où l'on est à l'école d'ingénieurs.

L'informatique semble devenir une maîtrise exigeante qui esquiverait les maîtres. Il faut qu'elle reste une maîtresse-servante déchargeant l'ingénieur des tâches ancillaires et suscitent un progrès constant dans la gestion intelligente des chiffres et le management du temps. Avec une giberne informatique bien faite et bien remplie, l'ingénieur hydraulicien est prêt à toutes les reconversions dès la sortie de l'Ecole ou plus tard.

La mécanique des fluides, c'est la signature génétique des rejetons de nos écoles d'hydraulique. Cet enseignement de la mécanique de fluides doit être moderne, complet, progressif.

Parce qu'il sera progressif et concret à la manière anglosaxonne, il sera dès la première année en Ecole utile et utilisable. Très vite, l'élève sera familiarisé avec le ballet de l'énergie sous la baguette de BERNOULLI et avec l'omniprésence du frottement visqueux ou déformation rhéologique, germe unique de la dissipation de la précieuse énergie.

Sur ces bases claires et stables dans l'esprit de l'étudiant, seront ensuite ancrées en deuxième niveau des notions plus délicates par exemple de la modélisation de la turbulence.

Puis viendra en dernière année, l'éducation de troisième cycle avec par exemple la mécanique des fluides numérique assimulant les développements les plus récents.

A côté de cet essentiel à la fois concret et théorique, à côté et non point à l'opposé sera l'existentiel qui groupera un ensemble de cours propres à développer la méthodologie de l'ingénieur mais qui ne sont pas chacun impératif dans la formation de l'ingénieur hydraulicien, pourront être à option ou changer d'une année à l'autre. Ils concernent des connaissances certes utiles à l'ingénieur mais qui pourraient être acquise par l'étudiant ou plus tard l'ingénieur sans l'intervention nécessaire de la structure pédagogique académique. Et la preuve est faite par le succès de

La philosophie est écrite dans ce très grand livre qui se tient constamment devant les yeux (je veux dire l'univers) mais elle ne peut se saisir si tout d'abord on ne saisit point de la langue et si on ignore les caractères dans lesquels elle est écrite. Cette philosophie est écrite en langue mathématique sans laquelle on ne fait qu'errer dans un labyrinthe obscur (Galilée). connaissance et d'application de certains étudiants réputés par leur absentéisme épisodique ou chronique de la voie autodidacte n'est pas forcément inefficace.

Ces cours pourront être de culture générale mais plutôt de spécialisation et de technologie : ce peut être par exemple le béton, l'hydrologie, les barrages, les chaudières, l'assainissement, les échangeurs, le traitement des eaux, l'isolation. Même disparates, leur choix en petit nombre doit permettre à l'élève ingénieur par le contact avec un homme de métier de se faire une idée précise et pratique de la méthodologie de l'ingénieur. Cette méthodologie consiste à bien identifier les étapes de l'activité intellectuelle et pratique de l'ingénieur dans la résolution d'un problème :

- mise en forme de la définition du problème et de l'objectif,

- recensement critique des modes de solutions possibles, - choix rationnel sous contraintes des moyens de résolution optimal (ou jugé tel)

- mise à disposition de l'utilisateur du résultat produit ou information avec diffusion d'un mode d'emploi adéquat simple, bref mais complet,

- suivi de l'usage du produit et réflexion critique sur son amélioration,

- archivage facilement accessible et utilisable de l'expérience acquise.

Ce cheminement, il ne s'agit pas de le suivre sur toutes les matières que pourrait rencontrer l'ingénieur hydraulicien dans sa future vie professionnelle mais bien de l'inculquer concrètement et profondément à chaque élève qui le personnalisera en le digérant à partir de quelques exemples. Il faut créer en lui une expérience, des mécanismes intellectuels, des réflexes qui assureront finalement l'efficacité sans fatigue.

A côté de cette initiation pratique et approfondie à la méthodologie de l'ingénieur grâce à deux ou trois matières, il faudra bien entendu multiplier les occasions de curiosité et de réflexion critique par des conférences, débats, discussions avec des professionnels qui parleront de leur vie.

Comme on le voit cette partie existentielle du programme sera encore plus souple et plus malléable que la partie essentielle. En très peu de temps, à très court terme, elle pourra s'adapter aux sollicitations de la profession et suivre le vent. Et tant pis si certains esprits chagrins traitent de girouette les manageurs pédagogiques. Comme disait Edgar FAURE « Ce n'est pas la girouette qui change, c'est le vent. "

A titre d'illustration, sur ces questions de nuance de formation, je donnerai la répartition des orientations, des travaux de diplôme des élèves ingénieurs hydrauliciens de l'ENSEEIHT au printemps 1990. Comme on peut le voir, sur la figure, la distribution entre tâches dominantes de recherche expérimentale $\mathrm{R}$, de code de calcul numérique $\mathrm{C}$ ou de méthodologie de l'ingénieur I, varie avec les options mais n'est-ce pas le signe que chaque étudiant peut y trouver son compte en fonction de sa personnalité et de ses objectifs d'avenir. Il faut de tout pour faire un monde même économique. Un spectre de formation trop étroit et figé serait un risque face aux surprises du futur. 


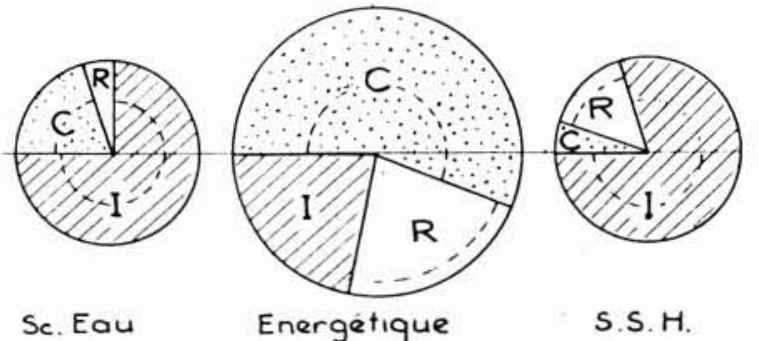

Sc. Eau
Energétique

S.S.H.
Domaine d'intérêt des diplomes d'ingénieurs 1990 (Département d'hydraulique)

I- Méthodologie de l'ingénieur

$C$ - Code de calcul

$R$ - Recherche expérimentale

\section{L'hydroludique ou la préparation à la civilisation des loisirs}

Petit préambule celui qui va écrire ces lignes a vraiment donné dans sa vie la part du lion au travail. Encore cette année, il a souvent passé dix à onze heures par jour pour concilier animation d'une équipe de recherche, direction d'un département pédagogique et relations internationales. Mais cet de cette expérience qu'il peut envisager un autre mode de vie pour ceux qui seront demain nos étudiants et après demain les ingénieurs (hydrauliciens ou non).

En définitive l'homme a un insidieux plaisir masochiste à s'aliéner lui-même. A une époque où en France, il suffit de $7 \%$ de la population aux champs pour gaver de nourriture tout l'hexagone, on s'est ingénié à créer des tâches inutiles pour assurer le mouvement brownien de la fourmilière salariée. Et la complexification de la vie est telle, les liens tissés si nombreux que beaucoup d'entre nous sont happés, déchiquetés par le rythme trépidant qu'ils contribuent à entretenir. Est-ce vraiment cette déshumanisation que l'on souhaite instaurer dans la vie de nos élèves dès l'école? La civilisation des loisirs serait-elle l'antidote ou bien simplement une nouvelle fuite en avant mercantile ? Depuis un an, je collectionne les coupures de presse traitant de près ou de loin des liaisons entre les métiers de l'eau et le monde des loisirs. Les titres sont significatifs : « Bourgogne, la Lyonnaise des eaux va ouvrir son premier parc de loisirs (Les Echos 3-11-89). " Le tourisme emploie 100 millions de personnes dans le monde " (de belles perspectives d'accroissement pour les six milliards d'hommes). "Salon nautique de Paris, retour en force du moto nautisme ", "Avec les canons à neige, la poudreuse sur commande ", "Rêves fluviaux à Montpellier-sur-Lez (Le Monde 23-1-90. " "Coup de soleil sur le Club Med (Nouvel Economiste 26-1-90. » " L'Aude aménage sa vallée », etc...

De temps immémoriaux, l'eau est présente dans le loisir : le bain, la pêche, l'aviron, la voile ou simplement la méditation poétique devant le charme discret du ruisseau oublié par la pollution ou la puisance majestueuse des cacades de Niagara ou d'Iguaçu.

Mais le jeu commence sans y paraître déjà au travail. La première activité hydroludique que je connaisse, c'est la recherche. Certes, en principe, elle est conventionnée pour le mieux de l'humanité mais dans quatre-vingt pour cent des cas, elle sert d'abord au plaisir du chercheur. Mais où est le mal ? La fin dernière de l'homme est le bonheur alors si le chercheur le trouve dans son travail, à condition que cela ne coûte pas trop cher à la communauté, l'objectif est rempli.

Quelques hauts lieux de l'hydroludique sont les congrès généralement situés dans des sites agréables (pourquoi pas au bord de la mer?), ils donnent aux hydrauliciens l'occasion de tourisme tonique et le plaisir de la communication dont celui de s'entendre parler. La gratification intellectuelle peut y être à forte dose: on apprend des choses intéressantes parfois même apparemment utiles.

Ainsi donc comme on le voit, l'hydroludique a déjà envahi notre vie. Pourquoi pas celles de nos étudiants? Il suffit que nous ayons la volonté de déveloper chez eux (et avec eux tout de même) leur PIB, entendons leur Pouvoir d'Imagination et de Bonheur. C'est bien connu et pas seulement de la Banque mondiale, plus le PIB est élevé, plus le pays est développé. Et l'on revient là, à l'organisation des structures pédagogiques, à la fonction de production pédagogique qui était la fonction de transfert dans notre analyse initiale du système.

Bien sûr, il ne faut pas rêver béatement, il ne s'agit pas de transformer en Club Med nos Ecoles (encore que chacune a sa petite réserve ludique, par exemple mon collègue, le Professeur Truchasson organise depuis des années un stage de voile qui joue beaucoup pour la cohésion de la promotion). Mais les occasions sont tout de même nombreuses de faire jouer intelligemment nos étudiants : des énoncés de devoir peuvent être stimulants comme des grilles de mots croisés, les activités d'expression orale comme les tables rondes contradictoires ont un aspect théâtral. A Sup Aéro à Toulouse, les élèves font des travaux personnels sur l'étude en soufflerie de maquette de la $2 \mathrm{CV}$ de l'étudiant économiquement faible, de l'aileron de planche à voile, de la portance des skis, de l'aérodynamisme du skieur enveloppé de bande Velcro pour diminuer la traînée, des performances du deltaplane. Et même, je me suis laissé dire que ces étudiants étudiaient très doctement les petites rugosités créées sur les balles de golf pour assurer le décollement de la couche limite et ainsi diminuer la traînée. 


\section{Conclusion}

Il en faudrait peu pour que notre réflexion sur la formation ne débouche sur des questions philosophico-économiques achoppant sur la trilogie travail-argent-bonheur qui pourrait aussi être vu sous le triplet travail-revenu-activité. L'essentiel c'est entendu, c'est le bonheur fortement lié à la gratification personnelle apportée par l'activité (dont la forme extrême mais pas forcément la moins gratifiante est le repos). Pourquoi dans les nations globalement riches, un meilleur pilotage politico-économique ne permettrait-il pas une meilleure répartition de l'activité entre les hommes et dans la vie de chaque homme pour éviter de pérenniser cette société duale a deux vitesses avec d'un côté des ingénieurs courant après le temps et de l'autre des chômeurs honteux de leur "loisir"?

L'objectif 2000 des enseignants qui sont aussi des citoyens, n'est-il pas des ingénieurs efficaces et heureux parce qu'ils auront été dans leurs écoles d'hydraulique des étudiants heureux et efficaces.
"Le bonheur est dans le pré Cours-y vite, cours- $y$ vite Le bonheur est dans le pré Cours-y-vite, il est passé. "

\section{Bibliographie}

BOUVARD M. (1989): La formation de l'ingénieur hydraulicien européen en l'an 2000. Tentative de définition des questions principales. Notes manuscrites, 9 pages.

BESSIẺRES A. (1990): Un formateur initial d'ingénieurs s'interroge sur les capacités demandées aux ingénieurs dans la vie professionnelle et les conditions de leurs acquisitions. 29 pages, INSA Toulouse.

Chappuis R. (1986) : La psychologie des relations humaines, Que sais-je 2287, PUF.

GoLDSCHID Metal (1989): La réussite professionnelle des ingénieurs et architectes. Rapport final FNRS. Chaire de Pédagogie Ecole Polytechnique Fédérale de Lausanne, 85 pages.

IlLıCH I. (1977): Le chômage créateur, Editions du Seuil.

JospIN L. (1989): Allocution devant l'Assemblée Nationale, 7 juin.

JoSPIN L. (1989) : Discours au SITEL, 18 octobre.

Linhart D., Malan A. (1990) : Fin de siècle, début de vie. Voyage au pays des $18-25$ ans. Syros alternative, 6 , rue Montmartre, 75001 Paris.

Lоноu R. (1969) : Essai d'une définition d'un corps d'enseignement sur l'aménagement des eaux, notes manuscrites, 23 septembre.

VAN DER BEKEN (1988) : Education in hydrology and water management. An outline for cooperation, Laboratoire of Hydrology Free University Brussels, 56 pages.

(1989): Principes pour une réflexion sur les contenus de l'enseignement. Rapport de la Commission présidée par Pierre Bourdieu et François Gros, mars, 12 pages. 